HUTP-03/A021

\title{
Constructing Gravitational Dimensions
}

\author{
Matthew D. Schwartz \\ Jefferson Laboratory of Physics, Harvard University, Cambridge, MA 02138 \\ email: matthew@schwinger.harvard.edu
}

\begin{abstract}
It would be extremely useful to know whether a particular low energy effective theory might have come from a compactification of a higher dimensional space. Here, this problem is approached from the ground up by considering theories with multiple interacting massive gravitons. It is actually very difficult to construct discrete gravitational dimensions which have a local continuum limit. In fact, any model with only nearest neighbor interactions is doomed. If we could find a non-linear extension for the Fierz-Pauli Lagrangian for a graviton of mass $m_{g}$ which does not break down until the scale $\Lambda_{2}=\sqrt{m_{g} M_{\mathrm{Pl}}}$, this could be used to construct a large class of models whose continuum limit is local in the extra dimension. But this is shown to be impossible: a theory with a single graviton must break down by $\Lambda_{3}=\left(m_{g}^{2} M_{\mathrm{Pl}}\right)^{1 / 3}$. Next, we look at how the discretization prescribed by the truncation of the KK tower of an honest extra diemsinon rasies the scale of strong coupling. It dictates an intricate set of interactions among various fields which conspire to soften the strongest scattering amplitudes and allow for a local continuum limit, at least at tree-level. A number of canditate symmetries associated with locality in the discretized dimension are also discussed.
\end{abstract}




\section{Introduction}

There are many compelling reasons to study discrete gravitational dimensions. The ultimate goal, of course, is to construct a space-time lattice which reproduces general relativity at low energies. A more practical application would be towards phenomenological extensions of the standard model. Here we use them to characterize what type of low energy effective theories might have arisen from the compactification of a continuous extra dimensional space. Until recently, the best approach to this problem seemed to be to take an extra dimensional model and work out the low energy theory by explicitly integrating out the extra dimensions. Because the KK tower of such theories can be truncated at very high energy without harming the low energy theory, any such model can be interpreted as a discrete theory space by a simple Fourier transform. So the question becomes: which theory spaces produce low energy effective theories with an extra dimensional interpretation? Because we know how to study such gravitational theory spaces directly [1], we now have a very general approach to the problem.

Normally, we would expect that a discrete extra dimension should look continuous for small enough lattice spacing. This is true for gauge theories, where any haphazardly constructed theory space that looks continuous at the linear level can be made to look continuous at the non-linear level if the discretization is taken sufficiently fine. That is, violations of unitarity from the gauge boson interactions can be pushed above the natural cutoff of the higher dimensional theory by simply shrinking the lattice spacing. In a recent paper [2], it was shown that the same simple intuition does not apply for discrete dimensions involving gravity. For example, the continuum limit of a discretization with only nearest neighbor hopping terms must have interactions, apparent at low energy, which are highly non-local in the extra dimension. The problem is that for gravity self-consistent effective field theory imposes a limit on how weak we can make the unitarity violating effects. In [2], the origin of this impediment was traced to the crazy scalar longitudinal mode of a massive graviton which propagates only after mixing with the transverse modes. It was also argued in [2] that the truncated KK theory of a single compact extra dimensional model does have a local continuum limit. However, no explanation was given about how the scalar longitudinal mode is dealt with from the point of view of the low energy effective theory.

In this paper, we begin to explore how to construct theory spaces from the ground up. We elaborate on the results of [1] and [2] and close the book on a number of issues left unresolved by those investigations. First, we attempt to improve the minimal nearest neighbor discretization by adding non-linear, but still nearest neighbor, interactions among the site gravitons. If there were a extension of the Fierz-Pauli Lagrangian for a massive graviton which controlled the dangerous scalar longitudinal mode, this could be replicated around the minimal model and the continuum limit would be drastically improved. In Section

3 we show that no such extension exists. In fact, we completely characterize all non-linear extensions Fierz-Pauli and show conclusively that a theory for a single graviton of mass $m_{g}$ must break down by $\left(m_{g}^{2} M_{\mathrm{Pl}}\right)^{1 / 3}$. Next, we explore a truncated KK theory for the case of 
a circle. We study the interacting Lagrangian of this theory in great detail and compute all of the strongest tree-level amplitudes. The most dangerous amplitudes involving the troublesome scalar longitudinal mode of the lightest massive graviton are softened by the exchange of heavier gravitons and the massless graviphoton. The radion also contributes, but not to the strongest diagrams. Finally, we make some comments about various broken symmetries and discuss some implications of this work. Much of the technical details are removed to Appendices $\mathrm{A}$ and $\mathrm{B}$; all of the important qualitative results are presented in the main text.

\section{Goldstone Bosons and the Minimal Discretization}

We begin with a review of [1] and [2], with a few added niceties. The theories we will consider all contain at least one massive graviton, and therefore involve Lagrangians of Fierz-Pauli [3] form:

$$
\mathcal{L}=M_{\mathrm{Pl}}^{2} \sqrt{g} R(g)+M_{\mathrm{Pl}}^{2} m_{g}^{2}\left(g_{\mu \nu}-\eta_{\mu \nu}\right)\left(\eta^{\mu \rho} \eta^{\nu \sigma}-\eta^{\mu \nu} \eta^{\rho \sigma}\right)\left(g_{\rho \sigma}-\eta_{\rho \sigma}\right)+\cdots
$$

The mass term explicitly breaks general coordinate invariance (GC) and leads to the propagation of the longitudinal modes of the graviton. It is helpful to project out these modes directly as separate fields which can be interpreted as the Goldstone bosons for the breaking of the GC symmetry. This is done by applying the coordinate transformation $x^{\alpha} \rightarrow y^{\alpha}(x)=x^{\alpha}+\pi^{\alpha}(x)$ to the Lagrangian. The dependence of the new Lagrangian on the Goldstone bosons $\pi^{\alpha}$ conveys all the effects of the broken symmetry. More explicitly, we apply the following replacement to (2.1):

$$
g_{\mu \nu} \rightarrow \tilde{g}_{\mu \nu} \equiv \frac{\partial y^{\alpha}}{\partial x^{\mu}} \frac{\partial y^{\beta}}{\partial x^{\nu}} g_{\alpha \beta}(y)=\left(x^{\alpha}+\pi^{\alpha}\right)_{, \mu}\left(x^{\alpha}+\pi^{\beta}\right)_{, \nu} g_{\alpha \beta}(x+\pi)
$$

It also follows that the Lagrangian which results from this replacement is generally coordinate invariant. After all, the $\pi^{\alpha}$ represent all the symmetry violating effects. Of course, $\pi^{\alpha}$ must transform non-linearly, but its transformation law is simply induced from the transformation of $y^{\alpha}$ and given in [2].

At this point, it is useful to expand the metrics around flat space $g_{\mu \nu}=\eta_{\mu \nu}+h_{\mu \nu}$ and the Goldstone bosons as $\pi^{\alpha}=\eta^{\alpha \mu} A_{\mu}$. Then, after an integration by pars, the Lagrangian (2.1) becomes:

$$
\mathcal{L}=\frac{1}{4} M_{\mathrm{Pl}}^{2}\left(-h_{\mu \nu, \alpha}^{2}+2 h_{\mu \nu, \mu}^{2}-2 h_{\mu \nu, \mu} h_{, \nu}+h_{, \mu}^{2}\right)+M_{\mathrm{Pl}}^{2} m_{g}^{2}\left(F_{\mu \nu}^{2}+2 A_{\mu, \nu} h_{\mu \nu}-2 A_{\mu, \mu} h\right)+\cdots
$$

The first part is the standard kinetic term for a massless graviton, and the second part contains the standard kinetic term for $A_{\mu}$. Note that if we had chosen a tensor structure for the mass term in (2.1) different from Fierz-Pauli, $A_{\mu}$ would have non-standard kinetic term signalling unitary violation at $\sim m_{g}$.

$A_{\mu}$ is an interacting vector boson, which for lack of any gauge symmetry, contains three propagating degrees of freedom. We can separate out its longitudinal mode, which corresponds 
to the scalar longitudinal mode of $h_{\mu \nu}$ by substituting $A_{\mu} \rightarrow A_{\mu}+\phi_{, \mu}$. This establishes an artificial $U(1)$ symmetry for which $\phi$ is the Goldstone boson. We will return to this symmetry in Section 3 . Using a more schematic notation, the Lagrangian becomes:

$$
\mathcal{L}=M_{\mathrm{Pl}}^{2} h \square h+M_{\mathrm{Pl}}^{2} m_{g}^{2}(A \square A+h \square \phi)+\cdots
$$

In this Lagrangian, $\phi$ only gets a kinetic term from mixing with $h$. Naturally, because $\phi$ always appears with two derivatives, the only way it could get a proper kinetic term is through mixing. Nevertheless, this feature is the source of all the bizarre features of massive gravitons discussed in $[1,2]$ and expounded here.

To study the interacting theory, we need to canonically normalize the fields: $h_{\mu \nu}^{c}=$ $M_{\mathrm{Pl}} h_{\mu \nu}, A_{\mu}^{c}=m_{g} M_{\mathrm{Pl}} A_{\mu}$, and $\phi^{c}=m_{g}^{2} M_{\mathrm{Pl}} \phi$. Thus, each interaction will have an associated scale which we can read directly off the Lagrangian. Because all the strong interactions, involving the Goldstone fields, come out of the mass term in (2.1), we can derive a general formula:

$$
m_{g}^{2} M_{\mathrm{Pl}}^{2} A_{\mu}^{n_{A}} \phi^{n_{\phi}} h^{n_{h}}=\Lambda_{\lambda}^{4-n_{A}-n_{\phi}-h_{h}} A_{\mu}^{c n_{A}} \phi^{c n_{\phi}} h^{c n_{h}}
$$

where

$$
\Lambda_{\lambda}=\left(m_{g}^{\lambda-1} M_{\mathrm{Pl}}\right)^{1 / \lambda}, \quad \lambda=\frac{3 n_{\phi}+2 n_{A}+n_{h}-4}{n_{\phi}+n_{A}+n_{h}-2}
$$

This implies, for example, that the strongest vertex is $\phi^{3}$ which has the scale $\Lambda_{5}=\left(m_{g}^{4} M_{\mathrm{Pl}}\right)^{1 / 5}$. The amplitude for a simple exchange diagram involving this vertex will grow as $\mathcal{A} \sim E^{10} / \Lambda_{5}^{10}$.

Incidentally, it may seem strange that the Lagrangian (2.1) should be based on $\sqrt{g} R$ when general coordinate invariance is explicitly broken by the mass term. But this partial GC symmetry guarantees that all the interactions coming from the $\sqrt{g} R$ term involve only transverse polarizations. If this were not true, and a term like $M_{\mathrm{Pl}}^{2} \partial^{2} h^{3}$ were present with arbitrary tensor structure, it would produce interactions of $\phi$ which a simple calculation shows get strong at $\Lambda_{7}$. So, the GC symmetry in the $\sqrt{g} R$ term, which has all the interactions in unitary gauge, actually raises the scale of strong coupling. While this is not a qualitative improvement, it does demonstrate that $\Lambda_{5}$ is not the lowest possible scale where a theory for single massive graviton based on Fierz-Pauli could break down. In fact, the whole point of introducing Goldstone bosons as a symmetry breaking effect is that we can start at $\Lambda_{5}$; the cancellation of the $\Lambda_{7}$ diagrams, which would be obscure in unitary gauge, is given for free.

We continue our review by looking at the minimal lattice explored in [2]. The theory space picture looks like:

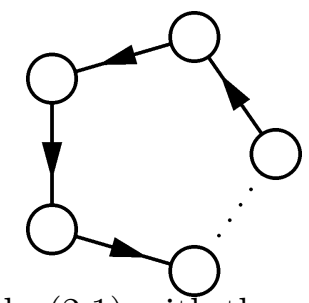

The associated Lagrangian is simply (2.1) with the mass terms replaced by hopping terms:

$$
\mathcal{L}_{\min }=\sum_{j} M^{2} \sqrt{g^{j}} R\left[g^{j}\right]+M^{2} m^{2} \sqrt{g^{j}}\left(g_{\mu \nu}^{j}-g_{\mu \nu}^{j+1}\right)\left(g_{j}^{\mu \rho} g_{j}^{\nu \sigma}-g_{j}^{\mu \nu} g_{j}^{\rho \sigma}\right)\left(g_{\rho \sigma}^{j}-g_{\rho \sigma}^{j+1}\right)
$$


The hopping terms break all but one of the general coordinate invariances. So we restore these symmetries by by replacing:

$$
g_{\mu \nu}^{j+1} \rightarrow \frac{\partial y_{j}^{\alpha}}{\partial x^{\mu}} \frac{\partial y_{j}^{\beta}}{\partial x^{\nu}} g_{\alpha \beta}^{j+1}\left(y_{j}\right)
$$

Next, we expand metrics around flat space and the $y_{j}$ in terms of vector and scalar Goldstones $a_{\mu}^{j}$ and $\phi^{j}$ (using lower case and $j$ for the site basis). Then the Lagrangian looks like:

$$
\mathcal{L}_{\text {min }}=M^{2} h_{j} \square h_{j}+M^{2} m^{2}\left\{\left(h_{j}-h_{j+1}\right)^{2}+\left(h_{j}-h_{j+1}\right) \square \phi_{j}+a_{j} \square a_{j}+\phi_{j} \phi_{j} \phi_{j}+\phi_{j} a_{j} a_{j}\right\}+\cdots
$$

To diagonalize the mass matrix, we take the standard linear combinations: $h_{j}=e^{2 \pi i \frac{j n}{N}} G_{n}$, $a_{j}=e^{2 \pi i \frac{j n}{N}} A_{n}$, and $\phi_{j}=e^{2 \pi i \frac{j n}{N}} \Phi_{n}$ (uppercase and $n$ for the momentum basis). Then, summing over $j$, and using the approximation $m_{n} \sim m \frac{n}{N}$ the Lagrangian becomes:

$$
\begin{aligned}
& \mathcal{L}_{\text {min }}=N M^{2} G_{n} \square G_{-n} \\
& +N M^{2} m^{2}\left\{\frac{n^{2}}{N^{2}} G_{n} G_{-n}+\frac{n}{N} G_{n} \square \Phi_{-n}+A_{n} \square A_{-n}+\Phi_{n} \Phi_{m} \Phi_{-n-m}+\Phi_{n} A_{m} A_{-n-m}+\cdots\right\}
\end{aligned}
$$

Just as with a single massive graviton, we can read of the strength of the interactions after going to canonical normalization: $G_{n}=\frac{1}{\sqrt{N} M} G_{n}^{c}, A_{n}=\frac{1}{\sqrt{N} M m} A_{n}^{c}$, and $\Phi_{n}=\frac{\sqrt{N}}{n M m^{2}} \Phi_{n}^{c}$. In terms of the the physical scales $M_{\mathrm{Pl}}=M \sqrt{N}$ and $m_{1}=m / N$ the strongest interactions look like:

$$
\mathcal{L}=\cdots+\frac{1}{N M_{\mathrm{Pl}} m_{1}^{4}} \Phi_{1}^{c} \Phi_{1}^{c} \Phi_{-2}^{c}+\frac{1}{N M_{\mathrm{Pl}} m_{1}^{2}} \Phi_{1}^{c} A_{1}^{c} A_{-2}^{c}+\cdots
$$

We then read off that the strong coupling scale, set by the $\Phi^{3}$ vertex is:

$$
\Lambda_{\min }=\left(N m_{1}^{4} M_{\mathrm{Pl}}\right)^{1 / 5}
$$

This scale seems reasonable. Formally, $\Lambda_{\text {min }}$ goes to $\infty$ as $N \rightarrow \infty$, and so we can reproduce linearized 5D gravity at low energy. However, within a consistent effective field theory, we can never take $\Lambda_{\text {min }}$ higher than the mass of the heaviest modes in the theory $m_{N} \sim N m_{1}$. This constraint, can be written as:

$$
\Lambda_{\min }<\Lambda_{\max }=M_{5 \mathrm{D}}\left(R M_{5 \mathrm{D}}\right)^{-5 / 8}
$$

where $R=1 / m_{1}$ is the size of the discrete dimension and $M_{5 \mathrm{D}}=\left(m_{1} M_{\mathrm{Pl}}^{2}\right)^{1 / 3}$ is the $5 \mathrm{D}$ Planck scale. Since $\Lambda_{\max }$ must be less than $M_{5 \mathrm{D}}$ this theory has no hope of looking like $5 \mathrm{D}$ gravity in the continuum limit.

Nevertheless, there is nothing wrong with taking $N \rightarrow \infty$ keeping $M_{\mathrm{Pl}}$ fixed in the minimal discretization. The resulting continuum theory will be a consistent effective field theory, even if it cannot be interpreted as having a smooth extra dimension. The argument in [2] for why the continuum limit will be non-local can be paraphrased as follows. The interactions in (2.10) are in terms of $\phi_{j}$, but $\phi_{j}$ gets a kinetic terms from coupling to $h_{j}-$ $h_{j+1}=\Delta_{z} h_{j}$. Equivalently, $\Phi=\Delta_{z} \phi_{j}=\phi_{j}-\phi_{j+1}$ is the physical, propagating field. So the dangerous interactions are really $\phi_{j}^{3} \sim \frac{1}{\Delta_{z}^{3}} \Phi^{3}$ which have a non-local continuum limit. 


\section{Improving the Minimal Model}

The simplest improvement on the minimal discretization would be a model which still only has nearest neighbor interactions, but whose unitary gauge Lagrangian is a more complicated function of $g_{\mu \nu}^{j}-g_{\mu \nu}^{j+1}$. These models are particularly easy to study, because all of their features can be understood from simply looking at non-linear extensions of the Fierz-Pauli Lagrangian for a single massive graviton. Of course, it is unlikely that this approach will provide a significant improvement over the minimal discretization, because these modifications are still strictly local, and we are trying to cure a non-local disease. Nevertheless, this fairly clean set of models will help us understand the locality problem within the low energy field theory. And if they were to succeed (which they won't) we would have all the freedom to construct gravitational theory spaces that we have for gauge theory spaces.

To begin, we should address the question of what property of the effective theory guarantees locality in the continuum limit. Recall that the obstacle to taking a smooth continuum limit of the minimal discretization is that all the modes are not necessarily lighter than the cutoff, a sine qua non of a consistent effective field theory. No such restriction exists for a weakly coupled gauge theory because the cutoff $\Lambda \sim 4 \pi N m_{1} / g$ is always above the top of the tower: $\Lambda>m_{N} \sim N m_{1}$. For gauge theory, the guarantee follows from the fact that $\Lambda$ depends on $N$ and $m_{1}$ only through the product $N m_{1} \sim m_{N}$. In contrast, for gravity the scale $\Lambda_{\text {min }}=\left(N m_{1}^{4} M_{\mathrm{Pl}}\right)^{1 / 5}$ does not depend on $N$ and $m_{1}$ in an auspicious combination. However, if we could find a Lagrangian for a single massive graviton which breaks down at $\Lambda=\Lambda_{2}=\sqrt{m_{g} M_{\mathrm{Pl}}}$ then the discretization based on this Lagrangian would have $\Lambda=\sqrt{N m_{1} M_{\mathrm{Pl}}}$ and would automatically satisfy the consistency constraint. This theory would have a local continuum limit. So our task becomes simply: extend the Lagrangian for a massive graviton so that it gets strong at $\Lambda_{2}$ (or higher).

Note, in passing, that the $\Lambda_{2}$ scale for a single massive graviton is the geometric mean between $M_{\mathrm{Pl}}$ and $m_{g}$. In particular, if we take the graviton to have a Hubble mass $m_{g} \sim H$, then $\Lambda_{2} \sim \mathrm{mm}^{-1}$, which happens to be the current limit to which gravity has been experimentally probed. Of course, the graviton could not have a Hubble mass because of other constraints from non-linear effects around large massive sources, as discussed at length in [1]. But if we look only at short distance constraints, raising the scale for strong coupling of a single massive graviton to $\Lambda_{2}$ would be absolutely necessary to avoid obvious contradiction with experiment.

Now, any Lagrangian we consider must start with a quadratic term of Fierz-Pauli form:

$$
\mathcal{L}_{\mathrm{FP}}=h_{\mu \nu}^{2}-h^{2}
$$

Since we already understand kinetic mixing, and are not presently interested in the relatively weakly coupled transverse modes, let us introduce the Goldstones as in (2.2) and then set $h_{\mu \nu}=0$. We will be making this transformation often for the rest of the paper and denote it $\rightsquigarrow$. It is equivalent to replacing:

$$
h_{\mu \nu} \rightsquigarrow A_{\mu, \nu}+A_{\nu, \mu}+A_{\alpha, \mu} A_{\alpha, \nu}
$$


Thus, after an integration by parts

$$
\mathcal{L}_{\mathrm{FP}} \rightsquigarrow-F_{\mu \nu}^{2}-4 A_{\mu, \mu} A_{\mu, \nu} A_{\mu, \nu}+4 A_{\mu \nu} A_{\nu \alpha} A_{\mu \alpha}+\cdots
$$

The appearance of $F_{\mu \nu}^{2}$ in (3.3) is suggestive. Recall that $\phi$, which we have not yet introduced into (3.3), is the longitudinal mode of the vector field $A_{\mu}$. It is the Goldstone boson for the breaking of a "fake" $U(1)$ symmetry which (3.3) has already at quadratic level. Of course, we cannot expect the entire Lagrangian to have a $U(1)$ symmetry, because $\phi$ is necessary to reproduce 5D gravity at the linear level. But we might hope that by adding cubic and higher order terms to (3.1), we can achieve a gauge invariance in the Goldstone Lagrangian, that is, the Lagrangian after the $\rightsquigarrow$ transformation. In other words, the FierzPauli structure may be the first part of an expansion fixed by $U(1)$ gauge invariance of the vector longitudinal modes. Moreover, we can see from (2.6) that all the interactions we are trying to get rid of, the ones which get strong below $\Lambda_{2}$, involve the field $\phi$. So this symmetry condition is sufficient for the construction of discretizations with local continuum limits.

Alas, it turns out that the $U(1)$ is a complete red-herring. We will now see not only that the $U(1)$ invariance embedded in the Fierz-Pauli structure is restricted to the quadratic

terms, but, more strongly, that there is no way to raise the scale of strong coupling for a single massive graviton higher than $\Lambda_{3}$.

\subsection{Extending Fierz-Pauli}

At this point, it is handy to introduce some notation. The vector of Goldstones, $A_{\mu}$, will always come with a derivative, so we can represent $A_{\mu, \nu}$ as a matrix:

$$
\begin{aligned}
A & \equiv A_{\mu, \nu} \Rightarrow A^{T}=A_{\nu, \mu} \\
F & \equiv A_{\mu, \nu}-A_{\nu, \mu}=A-A^{T} \\
\Phi & \equiv \phi_{, \mu, \nu}=\Phi^{T} \\
1 & \equiv \eta_{\mu \nu}
\end{aligned}
$$

Projecting out the longitudinal modes from a given unitary gauge Lagrangian amounts to replacing:

$$
h_{\mu \nu} \rightsquigarrow A+A^{T}+A^{T} A
$$

Also, since, by Lorentz invariance, we will always be taking traces of such matrices, we define the $[\cdots]$ notation by:

$$
[A \cdots A] \equiv \operatorname{Tr}[A \cdots A]=A_{\mu, \nu} \cdots A_{\alpha, \mu}
$$

So, in the new notation, the Fierz-Pauli term becomes:

$$
\begin{aligned}
\mathcal{L}_{\mathrm{FP}} & =\left[h^{2}\right]-[h]^{2} \\
& \rightsquigarrow\left[\left(A+A^{T}+A^{T} A\right)^{2}\right]-\left(2[A]+\left[A A^{T}\right]\right)^{2} \\
& =2\left[A A^{T}\right]+2\left[A A^{T}\right]-4[A]^{2}+4\left[A^{2} A^{T}\right]-4[A]\left[A A^{T}\right]+\left[A^{T} A A^{T} A\right]-\left[A A^{T}\right]^{2}
\end{aligned}
$$


The third line involved an integration by parts. We can express this in terms of the symmetric and antisymmetric parts of $A$ : $\Phi$ and $F$. That is, we set $A=\Phi+F$ and $A^{T}=\Phi-F$. Then, $\mathcal{L}_{\mathrm{FP}} \rightsquigarrow-4\left[F^{2}\right]+4\left[\Phi^{3}\right]-4[\Phi]\left[\Phi^{2}\right]+\left[F^{4}\right]-\left[F^{2}\right]^{2}+\left[\Phi^{4}\right]-\left[\Phi^{2}\right]^{2}-4\left[\Phi^{2} F^{2}\right]+2[\Phi F \Phi F]+2\left[F^{2}\right]\left[\Phi^{2}\right]$

With this notation, it will be much easier to study extensions of Fierz-Pauli. The $U(1)$ symmetry we are searching for implies that the Goldstone Lagrangian should depend only on $F$ and not on $\Phi$. First, observe that

$$
h \rightsquigarrow 2 \Phi+\Phi^{2}+\Phi F-F \Phi-F^{2}
$$

Since the only first order term in the expansion of $h$ is $\Phi$, we can always eliminate the $\Phi$ self-couplings from the Lagrangian. For example, we cancel the third order terms by adding

$$
\mathcal{L}_{3}=-\frac{1}{2}\left[h^{3}\right]+\frac{1}{2}[h]\left[h^{2}\right]
$$

Thus the lowest order $\Phi$ self couplings in $\mathcal{L}_{F P}+\mathcal{L}_{3}$ will be $\Phi^{4}$. These can be eliminated by adding an $\mathcal{L}_{4}$ with quartic terms, and so on. By induction, it is easy to see that all the self-interactions of the scalar can be eliminated in this way.

The next order gauge-violating terms look like $F^{2} \Phi^{2}$. Up to fourth order, there are 12 terms we must eliminate:

$$
\left[\Phi^{3}\right],[\Phi]^{2}[\Phi],[\Phi]^{3},\left[\Phi^{4}\right],\left[\Phi^{3}\right][\Phi],\left[\Phi^{2}\right]^{2},\left[\Phi^{2}\right][\Phi]^{2},[\Phi]^{4},\left[F^{2} \Phi^{2}\right],[F \Phi F \Phi],\left[F^{2}\right]\left[\Phi^{2}\right],\left[F^{2}\right][\Phi]^{2}
$$

These are related by two equations that come from integration by parts:

$$
\left[\Phi^{3}\right]=2[\Phi]^{2}+[\Phi]^{3} \quad \text { and } \quad\left[\Phi^{4}\right]=[\Phi]^{3}[\Phi]+\left[\Phi^{2}\right]\left[\Phi^{2}\right]+\left[\Phi^{2}\right][\Phi]^{2}+[\Phi]^{4}
$$

So there are 10 independent terms which must vanish. However, the most general Lagrangian up to fourth order in $h$ has only 8 terms:

$$
\mathcal{L}_{\Delta}=c_{1}\left[h^{3}\right]+c_{2}\left[h^{2}\right][h]+c_{3}[h]^{3}+q_{1}\left[h^{4}\right]+q_{2}\left[h^{2}\right]\left[h^{2}\right]+q_{3}\left[h^{3}\right][h]+q_{4}\left[h^{2}\right][h]^{2}+q_{5}[h]^{4}
$$

We might also consider terms with derivatives acting on $h$, but these cannot produce terms of the form (3.13). Therefore, unless there is some special arrangement, we do not have enough freedom to fabricate a $U(1)$ symmetry.

Still, it may be possible that although the Lagrangian is gauge dependent, all the physical scattering processes involving the $\phi$ fields vanish. This could be understood as a non-linearly realized $U(1)$ symmetry which is obscured by our choice of the transverse and longitudinal modes of $A_{\mu}$. For example, the field redefinition $A_{\mu} \rightarrow B_{\mu}+B_{\beta} B_{\mu, \beta}$ will produce interactions in the non-interacting Lagrangian $\left(A_{\mu, \nu}-A_{\nu, \mu}\right)^{2}$. These interactions will not vanish by integration by parts, but all the physical scattering amplitudes involving the new $B_{\mu}$ fields will be zero. We could certainly try to classify all non-linear field redefinitions, and all other reasons that the amplitudes may vanish while the interactions do not. But it is more straightforward just to compute the dependence of the strongest scattering amplitudes on the coefficients in (3.15). This is done in Appendix A. The conclusion is that it is simply impossible to extend the Fierz-Pauli Lagrangian so that unitarity is preserved for a single massive graviton above $\Lambda_{3}$. 


\section{Truncated KK Theory}

We have seen that it is impossible to eliminate all the dangerous amplitudes for scattering of the scalar longitudinal modes of a massless graviton by a non-linear extension of the FierzPauli Lagrangian. Had this been possible, we could have used the extended Lagrangian as a template for the link structure in a discretization with only nearest neighbor interactions, and the resulting theory would have had none of the problems of the minimal model discussed in [2]. This failure is disappointing, but could have been anticipated from the fact that the continuum sickness of the minimal model is non-locality in the extra dimension, so its cure should involve non-locality on the lattice.

Despite the discouraging results of Section 3, we know that the scale of strong coupling for a discrete extra dimension can be raised above $\Lambda_{\min }=\left(N m_{1}^{4} M_{\mathrm{Pl}}\right)^{1 / 5}$. As was mentioned in [2], a truncated KK theory does have a local continuum limit, in contrast to the minimal discretization. This simply follows from the observation that a truncation performed at high energy cannot effect low energy physics without undermining the general assumptions of effective field theory. Somehow the low KK modes are not interacting strongly at $\Lambda_{5}$ or even at $\Lambda_{3}$.

In this section, we study the truncated KK theory in great detail. We work out the Lagrangian in theory space, including the radion, graviphoton, and all the tensor structure. Its non-locality, including the power-law decay of the interactions with distance in the discrete dimension, is apparent. There are a number of subtle issues about the introduction of Goldstone bosons which are also explored. Finally, the amplitudes for all the dangerous diagrams involving the scalar longitudinal polarizations of the lowest KK modes are given. Due to exchange of heavier modes, and, somewhat surprisingly, the radion and graviphoton as well, all the dangerous amplitudes cancel at tree level.

Start with a 5D metric $G_{M N}$. We will label the compact fifth direction as $z$ and the noncompact directions collectively as $x$. Then we gauge fix as much as possible, so the metric takes the form

$$
g_{5 D}=\left(\begin{array}{cc}
g_{\mu \nu}(x, z)+e^{2 r(x)} V_{\mu}(x) V_{\nu}(x) & e^{2 r(x)} V_{\mu}(x) \\
e^{2 r(x)} V_{\nu}(x) & e^{2 r(x)}
\end{array}\right)
$$

$r$ is the radion and $V_{\mu}$ is the graviphoton. In this gauge, neither $r$ nor $V_{\mu}$ depends on $z$ and the Lagrangian becomes:

$$
\begin{aligned}
& \mathcal{L}= M_{5 \mathrm{D}}^{3} \sqrt{g_{5 D}} R_{5 D}\left(g_{5 D}\right) \\
&= M_{5 \mathrm{D}}^{3} \sqrt{g(x, z)}\left\{e^{r} R_{4 D}(g)+\frac{1}{4} e^{-r}\left(\partial_{z} g_{\mu \nu}\left(g^{\mu \rho} g^{\nu \sigma}-g^{\mu \nu} g^{\rho \sigma}\right) \partial_{z} g_{\rho \sigma}\right)\right. \\
&\left.-\frac{1}{4}\left(V_{\mu, \nu}-V_{\nu, \mu}\right) g^{\mu \nu} g^{\rho \sigma}\left(V_{\rho, \sigma}-V_{\sigma, \rho}\right)+\mathcal{L}_{V}\right\}
\end{aligned}
$$

Here $R_{5 D}\left(g_{5 D}\right)$ is the five dimensional Ricci scalar constructed from 5D metric. $R_{4 D}(g)$ is the $4 \mathrm{D}$ Ricci scalar constructed out of $g_{\mu \nu}(x, z)$ which we treat as a $4 \mathrm{D}$ metric which happens to depend on a continuous parameter $z \cdot \mathcal{L}_{V}$ contains interactions of the graviphoton with the other fields, some of which are presented in Appendix B. 


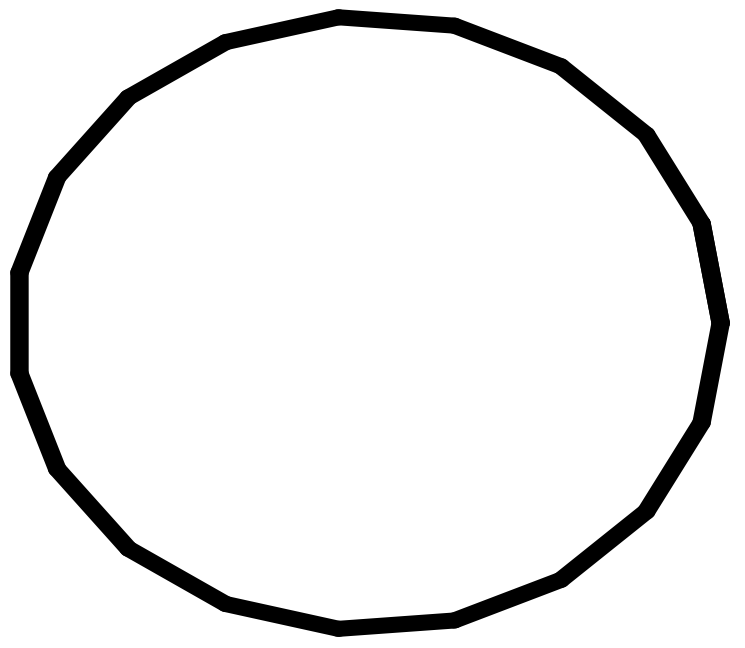

minimal discretization

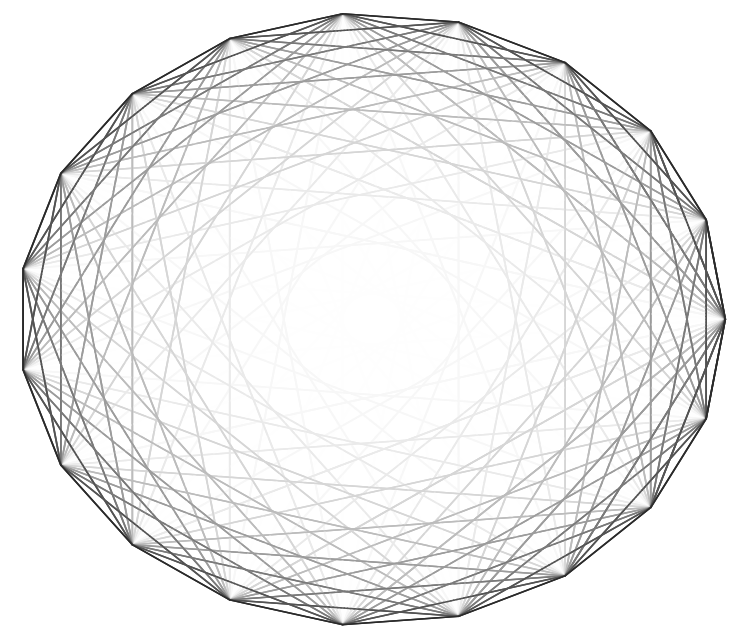

truncated KK theory

Figure 1: In the minimal deconstruction links are only between nearest neighbors. In the site basis for the truncated KK theory, there are links between every pair of sites, but the strength of the link dies off with distance. These links which are non-local in theory space remain in the limit of a large number of sites.

Now, we assume the compact dimension is a circle and expand the metric in KK modes: $g_{\mu \nu}(x, z)=\sum G_{\mu \nu}^{j}(x) e^{2 \pi i \frac{j z}{R}}$. We continue the convention that lower-case fields are in the site basis and uppercase fields are in the KK basis. Then $\mathcal{L}$ contains the mass terms:

$$
\mathcal{L}=M_{\mathrm{Pl}}^{2} m_{1}^{2} n^{2}\left(\left[G_{n} G_{-n}\right]-\left[G_{n}\right]\left[G_{-n}\right]\right)+\cdots
$$

The scale for the masses is set by the radius: $m_{1}=2 \pi / R$; and we have introduced the effective $4 \mathrm{D}$ Planck scale of the low energy theory: $M_{\mathrm{Pl}}^{2}=M_{5 \mathrm{D}}^{3} R$. We see that the spectrum comprises a massless graviton, a doubly degenerate tower of massive gravitons, and the massless radion and graviphoton. ${ }^{1}$

Now we truncate the theory to $N$ modes, and go back to position space via the discrete Fourier transform $G_{n}=\frac{1}{N} e^{2 \pi i \frac{n a}{N}} g_{a}$. Then, the mass terms become:

$$
\mathcal{L}_{2}=n^{2} G_{n} G_{-n}=\frac{n^{2}}{N^{2}} e^{2 \pi i n \frac{\Delta a}{N}} g_{a} g_{a+\Delta a}=\frac{2 n^{2}}{N^{2}} \cos \frac{2 \pi n \Delta a}{N} g_{a} g_{a+\Delta a}
$$

Evidently, there are links between distant sites. As $N \rightarrow \infty$

$$
\mathcal{L}_{2} \rightarrow \frac{1}{6 \pi^{2}} N\left(2 g_{a}^{2}-\frac{1}{\Delta a^{2}} g_{a} g_{a+\Delta a}\right)
$$

\footnotetext{
${ }^{1}$ It might seem that the theory would have been simpler if we had compactified on an interval instead of a circle, thereby removing the mass degeneracy and the graviphoton. In fact, because the KK wavefunctions for the interval are sines instead of exponentials, the interactions in the circle are much easier to work with. This point is discussed further in Appendix B.
} 
Even in this limit, the interactions which are non-local in theory space are only power-law suppressed. This is contrasted to the case of the minimal discretization in Figure 1 .

Now let us look at the interactions. We know that in a continuum limit, the theory would break down at the 5D Planck scale $M_{5 \mathrm{D}}=\Lambda_{3 / 2}=\left(m_{1} M_{\mathrm{Pl}}^{2}\right)^{1 / 3}$. We also know that if we truncate to two modes, a massless and a massive one, the theory would break down at least by $\Lambda_{3}=\left(m_{1}^{2} M_{\mathrm{Pl}}\right)^{1 / 3} \ll \Lambda_{3 / 2}$. This is due to scattering of the first massive mode, with mass $m_{1}$. So the additional modes must somehow cancel the strongest diagrams involving mode 1. Moreover, we know which fields may contribute to this cancellation. Momentum conservation in the fifth direction translates to KK number conservation in the $4 \mathrm{D}$ theory. So the only fields which can contribute to tree-level scattering of the gravitons at the first mass level are gravitons at the second mass level (with mass $m_{2}=2 m_{1}$ ), the massless graviton, the graviphoton, and the radion. Therefore, we are interested in the tree level processes:

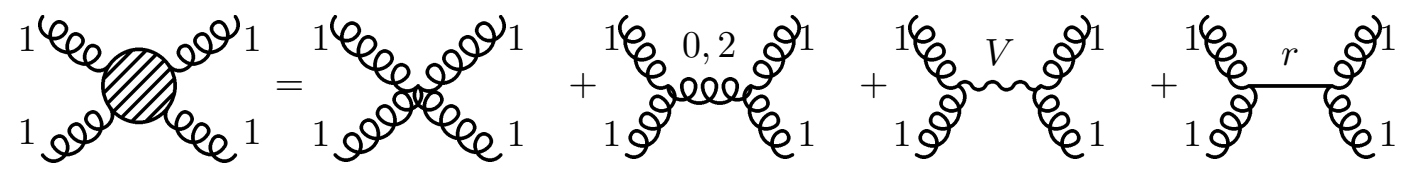

The Goldstone boson formalism lets us analyze the diagrams in (4.8) very efficiently. We can classify the amplitudes according to their energy dependence, i.e. whether they get strong at $\Lambda_{5}, \Lambda_{4}, \Lambda_{3}$, etc.. Because of the result from Section 3, that a single massive graviton must break down by $\Lambda_{3}$, it is illuminating to see how the $\Lambda_{3}$ and stronger diagrams in the truncated KK theory cancel. This is demonstrated in explicit detail in Appendix B. As a brief summary, we find that there are no vertices that contribute to $\Lambda_{5}$; the quartic vertices which contribute to $\Lambda_{4}$ are canceled by exchange of the graviphoton; And both the graviphoton and the vector polarization of mode 2 are necessary to cancel the $\Lambda_{3}$ diagrams involving external vectors. This is very important, because it implies that we are very unlikely to find a compactification in which the second lightest massive KK mode is parametrically heavier than the lightest one. It also implies that the first tree level diagrams which the truncation affects involve the $\Lambda_{3}$ scale appropriate external modes at mass level $N / 2$. That is, the truncated KK theory gets strong at $\Lambda \sim\left(N^{2} m_{1}^{2} M_{\mathrm{Pl}}\right)^{1 / 3}$. It is worth pointing out that the calculations in the appendix are a highly non-trivial check on the goldstone boson equivalence theorem for gravity and the entire effective field theory for massive gravitons.

\section{Discussion and Outlook}

In this paper, a number of important features of theories with massive gravitons have been analyzed. An arbitrary theory space which has only nearest neighbor interactions will lead, in the continuum limit, to a theory which looks extra-dimensional at the linear level, but has highly non-local interactions. This was understood to be a result of the anomalously strong scattering amplitudes for the light gravitons in the theory. We attempted to eliminate these amplitudes with a non-linear extension of the Fierz-Pauli Lagrangian, but found that no such extension could push the strong coupling scale above $\Lambda_{3}$. We would need to push it as high as 
$\Lambda_{2}$ to improve the continuum limit of nearest neighbor discretizations. In contrast, a theory space based on the truncation of a KK tower coming from an honest extra dimension does have a local continuum limit. We saw that the scale for the scattering of the light KK modes was pushed above their $\Lambda_{3}$ through the exchange of additional massive gravitons and the massless graviphoton. The truncated KK theory breaks down at the $\Lambda_{3}$ scale of the modes whose tree-level scattering is affected by the truncation, namely those halfway up the tower.

The truncated KK theory gives us much insight into what theory spaces might come from an extra dimensional compactification. We saw that exchange of gravitons on the second mass level canceled strong four-point interactions of gravitons on the first mass level. So it is unlikely that any compactification will have a KK tower with the second lightest graviton parametrically heavier than the first. More generally, we should not expect to see a parametrically large mass gap between any set of modes. It would be interesting to see what happens on AdS backgrounds, where the strong coupling problem is ameliorated [1] and there is evidence that at least one graviton mode can be made extremely light $[4,5]$. Also, note that the radion was not necessary for cancellation of the strongest diagrams, and in fact couples with the same strength as the transverse graviton modes. Although it contributes to cancel amplitudes which blow up at $\Lambda_{3}<M_{5 \mathrm{D}}=\Lambda_{3 / 2}$, we have some freedom to manipulate the radion in the effective theory. For example, simply giving the radion a mass by hand will only affect diagrams at the $\Lambda_{2}$ level applied to the lowest mode. We did not explore the radion interactions in depth, and in fact it may be necessary to perform a wave-packet analysis to understand these sub-dominant amplitudes.

Incidentally, the significance of the $\Lambda_{3}$ scale is not at all clear. It seems to be more fundamental than the $\Lambda_{5}$ scale, which as was pointed out in Section 2 is only significant in the somewhat contrived Fierz-Pauli Lagrangian. In contrast, $\Lambda_{3}$ appears as the ultimate upper limit on the strong coupling scale of a theory with a single massive graviton. It is even more intriguing that in a theory constructed to have this strong coupling scale, the natural size for all the operators in unitary gauge is set by $\Lambda_{3}$ and dimensional analysis, a particularly clean situation (see equation (4.40) of [1]). Also, $\Lambda_{3}$, applied to the middle modes, determines the scale where the truncated KK theory breaks down. And we saw that it is the strongest scale to which exchange of transverse polarizations of the KK modes, and the radion, can contribute. However, $\Lambda_{3}$ is not enough to use for the construction of nearest neighbor discretizations which are guaranteed to be local in the continuum limit. Certainly, one important area for future investigation is the apparent coincidence of these various results.

Returning to the main theme of this paper, it would be nice if the truncated KK theory had some exact symmetry that the minimal discretization lacked. The natural candidate is the general coordinate reparameterization of $z$. But because $z$ is the discretized dimension, this acts on KK towers as the Virasoro algebra, which does not have any $N$-dimensional representations. So the symmetry is broken in both theories. In contrast, the GC symmetries of the non-discretized dimensions act faithfully on fields in the site basis. Of course, these GC symmetries are a fake, broken in unitary gauge, and re-established formally with the Goldstone bosons. But we cannot add Goldstone bosons to restore the Virasoro symmetry 
in either model because the fields belong to a truncated infinite dimensional multiplet. The $U(1)$ symmetry for which the $\phi$ fields are Goldstone bosons is also irrelevant. We saw in Section 3 that it cannot be made exact by extending Fierz-Pauli. Moreover, we know the $\phi$ fields must exist in any discretization because we need all the polarizations of the 4D massive gravitons to get the five propagating modes of a massless graviton in 5D. So the truncated KK theory does not seem to have any extra exact symmetries at all.

In fact, there is nothing fundamentally better about the truncated KK theory than the minimal discretization. Both provide consistent low energy effective theories. While locality in the fifth dimension seems nice, there is certainly no experimental evidence to support it. And from the model building point of view, it is likely that there are applications of gravity in theory space for which locality is just irrelevant. Recall that the theory space technology was originally developed in gauge theory to reproduce the phenomenology of an extra dimension [6,7], but it was soon seen to be well-adapted for the construction of models with a naturally light "little" Higgs [8]. Similarly, gravitational theory spaces may produce applications which have no extra dimensional interpretation at all.

Nevertheless, locality goes hand in hand with improved UV properties. We have seen this already in Section 2 where non-locality in the minimal discretization was traced to a low cutoff in the effective theory. And certainly part of the motivation for trying to construct gravitational dimensions comes from string theory, which is both local and UV finite. While the nearest neighbor discretizations have all the exact symmetries of the truncated KK theories, the latter seem to have qualitatively superior UV properties. Now that we understand the appropriate issues, we can work towards establishing a more precise relation between apparent locality and a higher cutoff. This may lead to a better understanding of quantum gravity, and perhaps even a new class of UV completions.

\section{Acknowledgements}

This work would not have been possible without the help of Nima Arkani-Hamed. I would also like to thank Howard Georgi for his insight, especially about the issues discussed in Section 3, Thomas Gregoire for assisting with many of the Feynman diagram calculations and Paolo Creminelli and Lisa Randall for helpful discussions.

\section{A. Raising the Scale for a Single Massless Graviton}

In this appendix it we show that a theory with a single massive graviton must break down by $\Lambda_{3}$. We demonstrate this by considering all possible extensions to the Fierz-Pauli Lagrangian and calculating of all the tree level amplitudes which must cancel. Because of subtleties with a possible non-linearly realized $U(1)$ invariance, this brute-force approach proves to be more convincing than possible symmetry based arguments.

Start with the Fierz-Pauli Lagrangian:

$$
\mathcal{L}_{\mathrm{FP}}=\sqrt{g} R(g)+\frac{1}{4}\left(\left[h^{2}\right]-[h]^{2}\right)
$$


The strongest diagrams generated by this Lagrangian blow up at $\Lambda_{5}$, but there are also diagrams which get strong at $\Lambda_{4}$ and $\Lambda_{3}$. More explicitly, for diagrams with four external lines, the $\Lambda_{5}$ is scalar scattering through scalar exchange; $\Lambda_{4}$ is scalar scattering; and $\Lambda_{3}$ is either vector scattering through scalar exchange or scalar vector scattering. In general, vector exchange contributes at the same order as the corresponding quartic vertex.

The only terms which may help cancel these tree-level scattering processes are cubic and quartic in $h$ :

$$
\Delta \mathcal{L}=c_{1}\left[h^{3}\right]+c_{2}\left[h^{2}\right][h]+c_{3}[h]^{3}+q_{1}\left[h^{4}\right]+q_{2}\left[h^{2}\right]\left[h^{2}\right]+q_{3}\left[h^{3}\right][h]+q_{4}\left[h^{2}\right][h]^{2}+q_{5}[h]^{4}
$$

It is not necessary to consider terms with space-time derivatives acting on $h$, because they contribute to amplitudes with different momentum dependence than the ones we are trying to cancel.

Now, we want to study the interactions coming from these terms. As usual, we do this by introducing Goldstone bosons, via $h \rightsquigarrow A+A^{T}+A^{T} A$ and invoking the equivalence theorem. We work at high energy, where any gauge dependent mass the Goldstone may have is irrelevant, and taken to be zero. This implies that $[A]=[\Phi]=0$ if these fields correspond to external lines (i.e. on-shell, massless particles). In particular, quartic terms which involve $[h]$ and cubic terms which involve $[h]^{2}$ do not contribute, at first order, to diagrams with four external longitudinal modes. So, $q_{3}, q_{4}, q_{5}$ and $c_{3}$ contribute only above $\Lambda_{3}$ and we can ignore them.

Thus, the interactions which may contribute up to fourth order in $\mathcal{L}_{\mathrm{FP}}+\Delta \mathcal{L}$ are:

$$
\begin{aligned}
& \mathcal{L}_{A}=\left(1+6 c_{1}\right)\left[A^{2} A^{T}\right]+\left(-1+4 c_{2}\right)[A]\left[A A^{T}\right]+2 c_{1}\left[A^{3}\right]+4 c_{2}\left[A^{2}\right][A] \\
&+2 q_{1}\left[A^{4}\right]+\left(6 c_{1}+8 q_{1}\right)\left[A^{3} A^{T}\right]+\left(3 c_{1}+4 q_{1}\right)\left[A A A^{T} A^{T}\right]+\left(\frac{1}{4}+3 c_{1}+2 q_{1}\right)\left[A A^{T} A A^{T}\right] \\
&+\left(-\frac{1}{4}+2 c_{2}+4 q_{2}\right)\left[A A^{T}\right]\left[A A^{T}\right]+\left(2 c_{2}+8 q_{2}\right)\left[A^{2}\right]\left[A A^{T}\right]+4 q_{2}\left[A^{2}\right]\left[A^{2}\right]
\end{aligned}
$$

The first processes we consider involve scalar exchange. Replacing $A \rightarrow \Phi$ once for each of the cubic terms in (A.3) shows that the relevant interactions are:

$$
\mathcal{L}_{A} \supset \phi\left(\left(1+6 c_{1}+8 c_{2}\right) A_{\nu, \alpha, \mu} A_{\mu, \alpha, \nu}+\left(-1+8 c_{2}\right) A_{\alpha, \nu, \mu} A_{\alpha, \mu, \nu}\right)
$$

We have integrated by parts to remove the derivatives from $\phi$. Amplitudes coming from these vertices are strong at either $\Lambda_{5}$, if the external $A_{\mu}$ are longitudinally polarized (i.e. $A_{\mu} \rightarrow \phi_{, \mu}$ ), or at $\Lambda_{3}$, if the external $A_{\mu}$ are transverse. Either way, the amplitude will be proportional to the scalar current in (A.4) squared and so (A.4) must exactly vanish. So $c_{2}=\frac{1}{8}$ and $c_{1}=-\frac{1}{3}$.

Next, we will look at the $\Lambda_{3}$ diagrams from the process $A A \rightarrow \Phi \Phi$, which get a contri- 
bution from vector exchange:

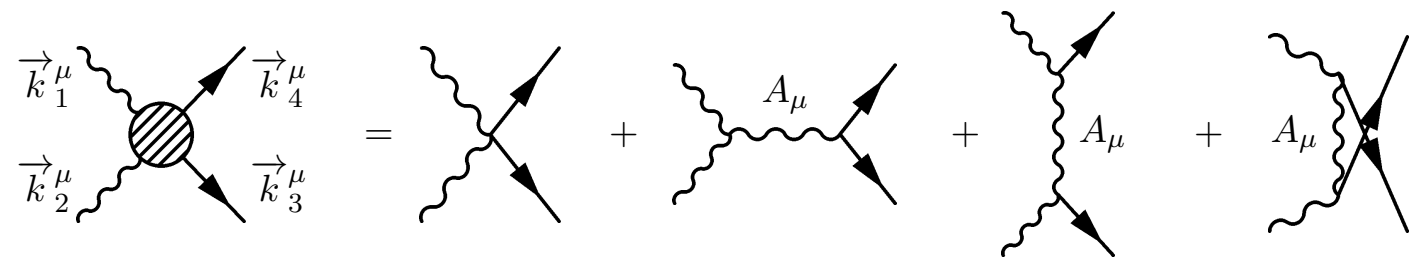

The total amplitude, with the values for $c_{1}$ and $c_{2}$ we just derived, is:

$$
\begin{aligned}
= & \left(4 q_{1}-1\right)\left[16\left(k_{3} \varepsilon_{1}\right)\left(k_{3} \varepsilon_{2}\right) t u+8\left(k_{1} \varepsilon_{2}\right)\left(k_{3} \varepsilon_{1}\right) t^{2}+8\left(k_{1} \varepsilon_{2}\right)\left(k_{3} \varepsilon_{1}\right) t u+8\left(k_{2} \varepsilon_{1}\right)\left(k_{3} \varepsilon_{2}\right) t u+8\left(k_{2} \varepsilon_{1}\right)\left(k_{3} \varepsilon_{2}\right) u^{2}\right] \\
& +\left(1+4 q_{1}+8 q_{2}\right)\left[8\left(k_{1} \varepsilon_{2}\right)\left(k_{2} \varepsilon_{1}\right)\left(t^{2}+u^{2}\right)\right]+\left(1+8 q_{1}+16 q_{2}\right)\left[-4\left(\varepsilon_{1} \varepsilon_{2}\right)\left(t^{3}+u^{3}\right)\right] \\
& +\left(3+4 q_{1}+24 q_{2}\right)\left[-8\left(\varepsilon_{1} \varepsilon_{2}\right)\left(t^{2} u+u^{2} t\right)+\left(3+16 q_{1}+32 q_{2}\right)\left[-4\left(k_{2} \varepsilon_{1}\right)\left(k_{3} \varepsilon_{2}\right)\left(t^{2}+u^{2}\right)\right]\right. \\
+ & \left(1+24 q_{1}+32 q_{2}\right)\left[-4\left(k_{3} \varepsilon_{1}\right)\left(k_{3} \varepsilon_{2}\right)\left(t^{2}+u^{2}\right)\right]+\left(3+24 q_{1}+32 q_{2}\right)\left[4\left(k_{1} \varepsilon_{2}\right)\left(k_{2} \varepsilon_{1}\right) t u\right] \quad(\mathrm{A} .5)
\end{aligned}
$$

We have used $k_{4}=k_{1}+k_{2}-k_{3}$ and the Mandelstam variables $s \equiv k_{1} k_{2}=k_{3} k_{4}, t \equiv k_{1} k_{4}=k_{2} k_{3}$ and $u \equiv k_{1} k_{3}=k_{2} k_{4}$. The terms are grouped to illustrate that no choice of $q_{1}$ and $q_{2}$ will make the entire amplitude vanish. Therefore, there is no Lagrangian for a single massive graviton which breaks down above $\Lambda_{3}$.

\section{B. Cancellations in Truncated KK Theory}

In this appendix we calculate the tree level scattering of the light graviton modes in a KaluzaKlein theory of 5D gravity. Along the way, some subtle issues about setting up the calculation and using the theory space formalism are addressed. In particular, we now defend the choice of a circle, which initially seems more complicated than the interval, because of the extra graviphoton degree of freedom and the degenerate spectrum. For a somewhat similar gauge theory calculation the reader is referred to $[9,10]$.

For a general compactification, the KK wavefunctions $\chi^{j}(z)$ depend on the the geometry and the boundary conditions. This lets the Lagrangian be expressed, before the truncation, as the infinite sum

$$
\mathcal{L}=\sum o_{i j}^{2} G_{i} G_{j}+o_{i j k}^{3} G_{i} G_{j} G_{k}+\cdots
$$

The $o^{n}$ are constants determined by overlap integrals of the $\chi^{j}$. For example, if we compactify on a circle of radius $R$, then $\chi_{n}(z)=e^{2 \pi i \frac{n z}{R}}$ and $o_{i j}=\int \chi_{i} \chi_{j} d z=\delta(i+j)$.

We now need a prescription for going from the KK basis to the theory space basis. That is, we need to choose discrete wavefunctions $g_{i}=c_{i j} G_{j}$. Of course, the obvious choice is a discrete Fourier sum, but we have to be very careful. The essential requirement for a nice theory space representation is that interactions which take place at fixed $z$ in the 5D theory (that is, those which do not depend on $\partial_{z}$ ) should transform to interactions which take place on a single site $j$. That way, the $R_{4 D}$ part of $\mathcal{L}$ (4.1) will have a separate general coordinate invariance for each site, and all the interactions of the longitudinal modes will 
come from the $\partial_{z}$ part. This has the advantage that all the interactions will be proportional to $M_{\mathrm{Pl}}^{2} \partial_{z}^{2} \sim M_{\mathrm{Pl}}^{2} m^{2}$ instead of $M_{\mathrm{Pl}}^{2} \partial^{2} \sim M_{\mathrm{Pl}}^{2} E^{2} \gg M_{\mathrm{Pl}}^{2} m^{2}$. The only way this will happen is if we can choose the discrete wavefunctions to have all the same overlap integrals as the continuous wavefunctions:

$$
o_{i \cdots k}=\frac{1}{R} \int_{0}^{R} d z \chi_{i} \cdots \chi_{k}=\frac{1}{N} \sum_{n} c_{i n} \cdots c_{k n}
$$

For a general KK theory this is not possible. But if we compactify on a circle, $\chi_{n}(z)=e^{2 \pi i \frac{n z}{R}}$, then $c_{a b}=e^{2 \pi i \frac{a b}{N}}$ satisfies (B.2). Of course, any KK theory has a theory space representation, but in general the theory space will not simplify the structure of the interactions. For example, it is not possible to satisfy (B.2) on an interval, and so there will be strong interactions simply from the sites' Lagrangians. While amplitudes involving these interactions must cancel other amplitudes at the end of the day, we get this cancellation for free if we choose to the circle where (B.2) holds.

\section{B.1 Circle Lagrangian}

The truncated KK theory on a circle has a degeneracy at each mass level. We will need the quadratic, cubic, and quartic parts of the KK Lagrangian which come from (4.3):

$$
\begin{aligned}
\mathcal{L} \supset & M_{\mathrm{Pl}}^{2} \mathcal{L}_{K}\left(G_{n} G_{-n}\right)-M_{\mathrm{Pl}}^{2} m_{1}^{2}\left[\delta(n+m) m n\left(\left[G_{n} G_{m}\right]-\left[G_{n}\right]\left[G_{m}\right]\right)\right. \\
& \left.+m n \delta(m+n+p)\left\{\frac{1}{2}\left[G_{p}\right]\left[G_{m} G_{n}\right]\right\}-2\left[G_{m} G_{p} G_{n}\right]+\left[G_{n}\right]\left[G_{p} G_{m}\right]+\left[G_{m}\right]\left[G_{p} G_{n}\right]\right\} \\
+ & \left.m n \delta(m+n+p+q)\left\{-\frac{1}{4}\left[G_{p} G_{q}\right]\left[G_{n} G_{m}\right]-\left[G_{n} G_{p}\right]\left[G_{m} G_{q}\right]+\left[G_{n} G_{p} G_{m} G_{q}\right]+2\left[G_{n} G_{p} G_{q} G_{m}\right]\right\}\right]
\end{aligned}
$$

The delta functions enforce KK number conservation, which is equivalent to momentum conservation in the fifth dimension. There are other cubic and quartic terms, but they are irrelevant for the scattering processes we consider, and so we omit them for clarity. $\mathcal{L}_{K}$ is the standard kinetic Lagrangian for a spin two field, as shown in (2.3), and has the same form for each KK mode.

At this point, we could Fourier transform (B.3) to the site basis. This is not hard, but the explicit form of the site Lagrangian is actually unnecessary for the calculations we are interested in. We know that in unitary gauge, the Lagrangian will have only one general coordinate invariance, under which all the $g_{j}$ transform as tensors. The $N-1$ broken GC symmetries correspond to the massive gravitons, whose longitudinal modes produce the strongest interactions in (B.3). To study these interactions, we introduce Goldstone bosons on each site in theory space. That is, we replace each site metric:

$$
g_{\mu \nu}^{j} \rightarrow \tilde{g}_{\mu \nu}^{j}=\frac{\partial y_{j}^{\alpha}}{\partial x^{\mu}} \frac{\partial y_{j}^{\beta}}{\partial x^{\nu}} g_{\alpha \beta}^{j}\left(y_{j}\right)
$$

This restores $N$ copies of general coordinate invariance to the Lagrangian. 
This brings up another subtle issue. The transformation properties of the $y_{a}$ are not uniquely determined, and different choices will actually result in different interactions among the Goldstones. Of course the scale of the strong interactions is the same for all choices, but the calculations of individual amplitudes may be very complicated if we do not introduce Goldstones in a judicious way. For now, we will make it so that the $\tilde{g}_{\mu \nu}^{a}$ are invariant under all $N$ general coordinate transformations. This ensures that the interactions among the Goldstones will respect a global translation invariance around the circle. The drawback of this choice is that the diagonal general coordinate invariance is now obscure and the Lagrangian seems to depend explicitly on $N$ sets of Goldstone bosons. Nevertheless, the Lagrangian must be independent of some non-linear combination of these Goldstone fields.

The $y^{\alpha}$ are then expanded in terms of Goldstone bosons as usual $y_{j}^{\alpha}=x^{\alpha}+a_{j}^{\alpha}$ (and, as usual, we use lower-case for the site basis). The Goldstones are introduced in the site basis, but immediately Fourier transformed: $a^{j}=e^{2 \pi i \frac{j n}{N}} A^{n}$. Since in the KK basis the masses are diagonal, the bilinear couplings of the Goldstones will be diagonal as well. The quadratic terms in the KK basis are:

$$
M_{\mathrm{Pl}}^{2}\left(n m_{1}\right)^{2}\left\{\left[\left(G_{n}+A_{n}+A_{n}^{T}\right)\left(G_{-n}+A_{-n}+A_{-n}^{T}\right)\right]-\left[G_{n}+2 A_{n}\right]\left[G_{-n}+2 A_{-n}\right]\right\}
$$

As with the single massive graviton case, the longitudinal modes of $A^{n}$ will pick up kinetic terms from mixing with $G^{n}$. Thus, the fields have canonical normalization as for a single graviton of mass $m_{n}=n m_{1}$.

The interactions we are interested in control the scattering of Goldstones. So after introducing the Goldstones in the site basis, we project out their interactions as in Section 3 by setting $g_{\mu \nu}^{j}=\eta_{\mu \nu}$. In the matrix notation (cf. (3.8) and (3.4)) this corresponds to:

$$
g^{j} \rightsquigarrow a^{j}+a^{j T}+a^{j T} a^{j}
$$

Because we have introduced the Goldstones on the sites, and they are therefore summed over, this implies

$$
G^{n} \rightarrow A^{n}+A^{n T}+A^{m T} A^{n-m}
$$

Thus, KK number will be preserved in Goldstone scattering.

We have glossed over another subtle point. Introducing Goldstone bosons on the sites is very different from assigning a general coordinate invariance to each KK mode $G_{\mu \nu}^{n}$. That would amount to replacing $G^{n} \rightsquigarrow A^{n}+A^{n T}+A^{n T} A^{n}$ which leads to interactions which violate KK number conservation. We are free to do this, and the physics will be exactly the same since both Lagrangians are the same in unitary gauge, but it will be much harder to calculate. Not only will we have introduced KK number violating interactions, but we will also have introduced interactions into the $R_{4 D}$ part of the Lagrangian, which as we noted before, can be canceled for free.

\section{B.2 Radion and Graviphoton}

The next step is to decide which of the massless fields have strong enough couplings to contribute to scattering of the massive KK gravitons modes. Since the radion, $r$, and the 
graviphoton, $V_{\mu}$, are massless, they get normalized with $M_{\mathrm{Pl}}$. Indeed, $V$ 's kinetic term is already present in $\mathcal{L}$ and the radion picks up a kinetic term from mixing with the massless zero-mode graviton. Heuristically,

$$
\begin{aligned}
\mathcal{L} & =M_{\mathrm{Pl}}^{2} \sqrt{G_{0}}\left\{R\left(G_{0}\right) e^{r}+\left(V_{\mu, \nu}-V_{\nu, \mu}\right)^{2}\right\}+\cdots \\
& =M_{\mathrm{Pl}}^{2} G^{0} \square G^{0}+M_{\mathrm{Pl}}^{2} r \square G^{0}+M_{\mathrm{Pl}}^{2}\left(V_{\mu, \nu}-V_{\nu, \mu}\right)^{2}+\cdots
\end{aligned}
$$

So canonical normalization is:

$$
V_{\mu}^{c}=M_{\mathrm{Pl}} V_{\mu} \quad \text { and } \quad r^{c}=M_{\mathrm{Pl}} r
$$

Independent of the detailed tensor structure, the couplings to the massive fields have the form:

$$
\mathcal{L}=\cdots+\frac{\partial^{2}}{M_{\mathrm{Pl}}} r^{c} A_{n}^{c} A_{n}^{c}+\frac{\partial^{3}}{M_{\mathrm{Pl}} m_{n}} V^{c} A_{n}^{c} A_{n}^{c}+\frac{\partial^{4}}{M_{\mathrm{Pl}} m_{n}^{2}} r^{c} \Phi_{n}^{c} \Phi_{n}^{c}+\frac{\partial^{5}}{M_{\mathrm{Pl}} m_{n}^{3}} V^{c} \Phi_{n}^{c} \Phi_{n}^{c}+\cdots
$$

We will study below the strongest contributions to tree-level scattering processes with each type of external lines. We can see from $(\overline{\mathrm{B} .10})$ that exchange of $r$ is a weaker process than exchange of the Goldstone vector $A_{\mu}$, while $V_{\mu}$ and $A_{\mu}$ exchange are the same strength. So, to first order, we can ignore $r$ but must include $V_{\mu}$. We will therefore need the interactions which are linear in $V_{\mu}$ and do not involve $r$ which were suppressed from the expression (4.4):

$$
\begin{array}{r}
\mathcal{L}_{V}=g^{\alpha \beta} g^{\mu \nu} g^{\gamma \delta} V_{\beta}\left(2 g_{\alpha \mu, z} g_{\nu \gamma, \delta}-g_{\alpha \mu, z} g_{\gamma \delta, \nu}-g_{\gamma \delta, z} g_{\alpha \mu, \nu}+\frac{1}{2} g_{\gamma \delta, z} g_{\mu \nu, \alpha}-\frac{3}{2} g_{\gamma \mu, z} g_{\delta \nu, \alpha}+g_{\gamma \mu, z} g_{\nu \alpha, \delta}\right) \\
+g^{\mu \nu} g^{\alpha \beta}\left(V_{\beta, \alpha} g_{\mu \nu, z}-V_{\beta, \mu} g_{\alpha \nu, z}\right)+g^{\mu \nu} V_{\beta}\left(2 g_{\mu \nu, \alpha, z}-2 g_{\alpha \mu, \nu, z}\right)+\cdots \quad \text { (B.11) }
\end{array}
$$

All of the couplings that we will need below come from the following terms in the KK decomposition of (B.11):

$$
\mathcal{L} \supset-i M_{\mathrm{Pl}}^{2} m_{1} n \delta(n+m) V_{\beta}\left(2 G_{\mu \nu}^{m} G_{\mu \nu, \beta}^{n}+G_{\mu \nu, \beta}^{m} G_{\mu \nu}^{n}-2 G_{\mu \nu}^{m} G_{\mu \beta, \nu}^{n}\right)
$$

Now let us come back to one of the subtle issues mentioned above. In addition to the radion and the KK gauge boson, we must contend with the zero mode Goldstone boson, $A_{\mu}^{0}$. It does not have a kinetic term, and does not pick one up by mixing. But it does have interactions, with the Lagrangian in its current form. The only reason we have this mode at all, is because when we introduced the Goldstone bosons in (B.6) we included one set for each of the $N$ sites, even though the Lagrangian has only $N-1$ broken coordinate invariances. The interactions of $A_{\mu}^{0}$ exist because the preserved symmetry is not the one under which all the fields on the sites transform nicely; it is the one where all the KK modes transform nicely. Suppose we had chosen the transformations of the Goldstone bosons so that each $\tilde{g}_{\mu \nu}^{j}$ were covariant under changes of a single coordinate $y^{s}$ and invariant under all the others. Then, $\tilde{g}_{\mu \nu}^{s}=g_{\mu \nu}^{s}$ and $\tilde{g}_{\mu \nu}^{j}=y_{j, \mu}^{\alpha} y_{j, \nu}^{\beta} g_{\alpha \beta}^{j}$ for $j \neq s$. Then there would be only $N-1$ sets of Goldstones 
and we would not have the peculiar $A_{\mu}^{0}$ field. The Lagrangian would be the same, but with $a_{\mu}^{s}=0$. In terms of the KK modes of the Goldstones, this implies

$$
A_{\mu}^{0}=-e^{2 \pi i \frac{s n}{N}} A_{\mu}^{n}
$$

For example, if we we take $s=0$ then $A_{\mu}^{0} \rightarrow-A_{\mu}^{1}-A_{\mu}^{2}-\cdots$. Now, there will be many KK number violating vertices. In particular, all the heavy KK modes would contribute to scattering of the low modes. But when we sum over all diagrams, KK number should not be violated. It would be nice if we could just use the residual general coordinate invariance to set $A_{\mu}^{0}=0$, but it not clear that this is consistent. There is no simple way of introducing Goldstones so that $A_{\mu}^{0}$ does not appear at all. As it turns out, if we calculate the scattering from terms generated by the substitution (B.13) into the cubic and quartic vertices involving the $A_{\mu}^{0}$, everything vanishes. This implies that we are probably free to just set $A_{\mu}^{0}=0$.

\section{B.3 Goldstone Interactions}

From now on, we use the notation $1 \equiv A_{\mu, \nu}^{1}$, where $A_{\mu}^{1}$ is the first KK mode of the vector of Goldstone bosons introduced. Scattering of the the two modes in the first massive level involves exchange of the two modes on the second massive level. So we need $N \geq 5$ to see a non-trivial cancellation. Therefore, we take $N=5$. Note that taking $N>5$ will not change the relevant vertices, as these get no contribution from higher KK modes. So we consider the lightest five modes $0,1,2,-1 \equiv \overline{1}$ and $-2 \equiv \overline{2}$, and restrict to diagrams involving only external 1 and $\overline{1}$. We will also immediately go to canonical normalization $A_{\mu}^{n} \rightarrow M_{\mathrm{Pl}} m_{n} A_{\mu}^{n}$ and $\Phi^{n} \rightarrow M_{\mathrm{Pl}} m_{n}^{2} \Phi^{n}$. Now, we present the relevant interactions among these fields.

We start with the cubic vertices. After a lengthy calculation we can isolate the following terms relevant for diagrams with external 1 and $\overline{1}$ :

$$
\begin{gathered}
\mathcal{L}_{C} \supset 8\left[11^{T} \overline{2}\right]-4\left[1^{T} 1 \overline{2}\right]+12[11 \overline{2}]+8\left[1^{T} 1^{T} \overline{2}\right]-14[11][\overline{2}]+2\left[1^{T} 1\right][\overline{2}] \\
-16[1][1 \overline{2}]-8[1]\left[1^{T} \overline{2}\right]+12[\overline{2}][1][1]+\text { c.c. }
\end{gathered}
$$

It is interesting to note that we can perform a field redefinition to remove all the cubic terms:

$$
2_{\mu} \rightarrow 2_{\mu}+\frac{1}{8} 1_{\beta} 1_{\mu, \beta}
$$

Note that this substitution preserves KK number conservation. This is an example of the type of non-linear transformation we were wary of in Section 3. But it does not actually make the calculations of this appendix any easier, so we leave $(B .14)$ as it is.

Next, the couplings of the graviphoton can be written as (from (B.12)):

$$
\mathcal{L} \supset 4 i V_{\beta}\left(1_{\mu, \nu} \overline{1}_{\beta, \mu, \nu}-\overline{1}_{\mu, \nu} 1_{\beta, \mu, \nu}\right)
$$

We have already dropped everything which vanishes when the Goldstones, which always appear on shell in $V$-exchange diagrams, are massless. The interactions in (B.16) contribute 
to 11 scattering at the order $\Lambda_{3}$, which is the same order as the quartic vertices and as 2 exchange.

Next, we look at the quartic vertices with 1 and $\overline{1}$ :

$$
\begin{aligned}
\mathcal{L}_{Q} \supset-8[\overline{11} 11]-4\left[1^{T} \overline{11} 1\right]+4\left[\overline{11} 1^{T} 1\right]-4\left[\overline{1}^{T} 11 \overline{1}\right]+4\left[\overline{1}^{T} \overline{1}_{11}\right] \\
-4\left[\overline{1}^{T} \overline{1}^{T} 11\right]+2\left[\overline{1}^{T} 1^{T} 1 \overline{1}\right]+2\left[1^{T} \overline{1}^{T} \overline{1} 1\right]-4[\overline{1} 1 \overline{1} 1]-4\left[\overline{1}^{T} 1^{T} \overline{1} 1\right]-4\left[\overline{1}^{T} 1 \overline{1} 1\right]-4\left[1^{T} \overline{1} 1 \overline{1}\right] \\
+6[11][\overline{11}]-[11]\left[\overline{11}^{T}\right]-\left[11^{T}\right][\overline{11}]+4[1 \overline{1}][1 \overline{1}]+4[1 \overline{1}]\left[1 \overline{1}^{T}\right]
\end{aligned}
$$

There are other terms involving [1] and [1]. These will not contribute when the external lines are on shell, so we have not displayed them here.

To calculate the interactions, it is easiest to project out the real and imaginary parts of the KK fields. This diagonalizes the kinetic terms of the physical fields. To account for the normalization as well, we make the substitution $1=\frac{1}{\sqrt{8}}(A+i B)$ and $2=\frac{1}{2 \sqrt{8}}(C+i D)$. Then the entire normalized Lagrangian we will need is:

$$
\begin{aligned}
& \mathcal{L} \supset-\frac{3}{16}\left[A^{4}\right]-\frac{1}{8}\left[A^{T} A^{3}\right]-\frac{1}{16}\left[A^{T} A^{T} A^{2}\right]+\frac{5}{32}\left[A^{2}\right]^{2}+\frac{1}{32}\left[A^{2}\right]\left[A A^{T}\right] \\
& -\frac{3}{16}\left[B^{4}\right]-\frac{1}{8}\left[B^{T} B^{3}\right]-\frac{1}{16}\left[B^{T} B^{T} B^{2}\right]+\frac{5}{32}\left[B^{2}\right]^{2}+\frac{1}{32}\left[B^{2}\right]\left[B B^{T}\right] \\
& -\frac{1}{8}[A B A B]+\frac{1}{8}\left[A^{T} B A B\right]+\frac{1}{8}\left[B^{T} A B A\right]+\frac{1}{8}\left[A^{T} B^{T} A B\right] \\
& -\frac{1}{4}\left[A^{2} B^{2}\right]-\frac{3}{8}\left[A^{T} A B B\right]-\frac{3}{8}\left[B^{T} B A A\right]-\frac{3}{16}\left[A^{T} B^{T} B A\right]-\frac{3}{16}\left[B^{T} A^{T} A B\right] \\
& +\frac{1}{8}\left[B^{T} A A B\right]+\frac{1}{8}\left[A^{T} B B A\right]+\frac{1}{8}\left[A^{T} A^{T} B B\right]-\frac{1}{16}\left[B^{2}\right]\left[A^{2}\right]+\frac{3}{8}[A B][A B] \\
& +\frac{3}{32}\left[A^{2}\right]\left[B B^{T}\right]+\frac{3}{32}\left[B^{2}\right]\left[A A^{T}\right]-\frac{1}{8}[A B]\left[A B^{T}\right] \\
& +\frac{1}{\sqrt{8}}\left[A A^{T} C\right]+\frac{1}{\sqrt{8}}\left[A^{T} A^{T} C\right]-\frac{1}{\sqrt{8}}[A A][C] \\
& -\frac{1}{\sqrt{8}}\left[B B^{T} C\right]-\frac{1}{\sqrt{8}}\left[B^{T} B^{T} C\right]+\frac{1}{\sqrt{8}}[B B][C] \\
& +\frac{1}{\sqrt{8}}\left[A B^{T} D\right]+\frac{1}{\sqrt{8}}\left[B A^{T} D\right]+\frac{1}{\sqrt{8}}\left[A^{T} B^{T} D\right]+\frac{1}{\sqrt{8}}\left[B^{T} A^{T} D\right]-\frac{1}{\sqrt{2}}[A B][D] \\
& -\frac{1}{2}\left[A^{T} A\right]+\frac{1}{2}\left[A^{2}\right]-\frac{1}{2}\left[B^{T} B\right]+\frac{1}{2}\left[B^{2}\right]-\frac{1}{2}\left[C^{T} C\right]+\frac{1}{2}\left[C^{2}\right]-\frac{1}{2}\left[D^{T} D\right]+\frac{1}{2}\left[D^{2}\right] \\
& -\frac{1}{4}\left(V_{\mu, \nu}-V_{\nu, \mu}\right)^{2}+V_{\beta}\left(A_{\mu, \nu} B_{\beta, \mu, \nu}-B_{\mu, \nu} A_{\beta, \mu, \nu}\right)
\end{aligned}
$$

\section{B.4 Test the Lagrangian}

To test the Lagrangian, we will look at some characteristic processes. Recall that the strength of a vertex for involving a graviton of mass $m_{g}$ is given by $\Lambda_{\lambda}=\left(m_{g}^{\lambda-1} M_{\mathrm{Pl}}\right)^{1 / \lambda}$ where $\lambda$ is 
given by (2.6). The strongest processes involve scalar exchange. And the vector exchange diagrams have the same strength as the diagrams coming from the quartic vertices as well as the diagrams with graviphoton exchange. If we separate out scalar exchange, which must cancel by itself, then the strength of a process is determined by the external lines. Since this is the case, we do not have even to project out the scalar in the external lines, we can just leave it as the longitudinal polarization of the vector field. We only insist that the polarization of the eternal vector field satisfies $\varepsilon_{\mu, \mu}=0$.

The masses of the Goldstone bosons are gauge dependent, but we will only be concerned with the lowest order tree level effects, and so we take all the Goldstones to be massless. The corrections, of order $m^{2} / k^{2}$ will only contribute to higher order processes which we will ignore. In practice, this means dropping terms which contain $[A],[B], A_{\mu, \mu}, B_{\mu, \mu}, A_{\mu, \nu, \nu}$ or $B_{\mu, \nu, \nu}$. We have already done this for the Lagrangian (B.17) but more simplifications come about for particular process.

The cubic terms' contribution to $C$ and $D$ exchange can be written as:

$$
\begin{aligned}
\mathcal{L} \supset & -\frac{1}{\sqrt{2}} C_{\mu}\left(A_{\alpha, \beta} A_{\mu, \alpha, \beta}-A_{\alpha, \beta} A_{\beta, \alpha . \mu}-B_{\alpha, \beta} B_{\mu, \alpha, \beta}+B_{\alpha, \beta} B_{\beta, \alpha, \mu}\right) \\
& -\frac{1}{\sqrt{2}} D_{\mu}\left(A_{\nu, \alpha} B_{\mu, \alpha, \nu}+A_{\mu, \alpha, \beta} B_{\beta, \alpha}-A_{\alpha, \beta, \mu} B_{\beta, \alpha}-A_{\alpha, \beta} B_{\beta, \alpha, \mu}\right)
\end{aligned}
$$

It is easy to see that for scalar exchange $C_{\mu} \rightarrow \phi_{, \mu}^{C}$ and $D_{\mu} \rightarrow \phi_{, \mu}^{D}$ the above terms vanish after integrating by parts. This means that all the scalar exchange processes, which are stronger than the corresponding vector exchange processes, vanish.

Next, consider the process $\phi^{A} \phi^{A} \rightarrow \phi^{B} \phi^{B}$. This contributes at the scale $\Lambda_{4}$. If we make the substitution $A_{\mu} \rightarrow \phi_{, \mu}^{A}$ and $B_{\mu} \rightarrow \phi_{, \mu}^{B}$ into (B.18) and (B.19) we see that there is no contribution from vector $C$ and $D$ exchange:

The quartic terms are:

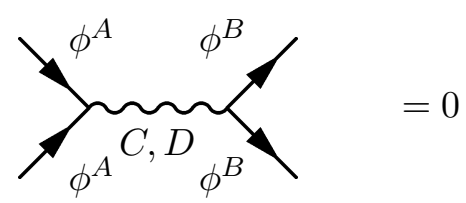

$$
\mathcal{L} \supset-\left[\Phi^{A} \Phi^{A} \Phi^{B} \Phi^{B}\right]+\frac{1}{4}\left[\Phi^{A} \Phi^{B} \Phi^{A} \Phi^{B}\right]+\frac{1}{4}\left[\Phi^{A} \Phi^{B}\right]\left[\Phi^{A} \Phi^{B}\right]+\frac{1}{8}\left[\Phi^{A} \Phi^{A}\right]\left[\Phi^{B} \Phi^{B}\right]
$$

This does not vanish by integration by parts. If we define the Mandelstam variables $s=p_{1}^{A} \cdot p_{2}^{A}$, $t=p_{1}^{A} \cdot p_{1}^{B}$ and $u=p_{1}^{A} \cdot p_{2}^{B}$ then this contributes:

$$
p_{2}^{A} p_{1}^{B}=-2 s^{2}\left(t^{2}+u^{2}\right)+t^{2} u^{2}+\frac{1}{2}\left(t^{4}+u^{4}\right)+\frac{1}{2} s^{4} \neq 0
$$

However, there is also a contribution from the graviphoton:

$$
\mathcal{L} \supset V_{\beta}\left(\phi_{, \mu, \nu}^{A} \phi_{, \beta, \mu, \nu}^{B}-\phi_{, \mu, \nu}^{B} \phi_{, \beta, \mu, \nu}^{A}\right)
$$


This contributes through the $t$ - and $u$ - channels:

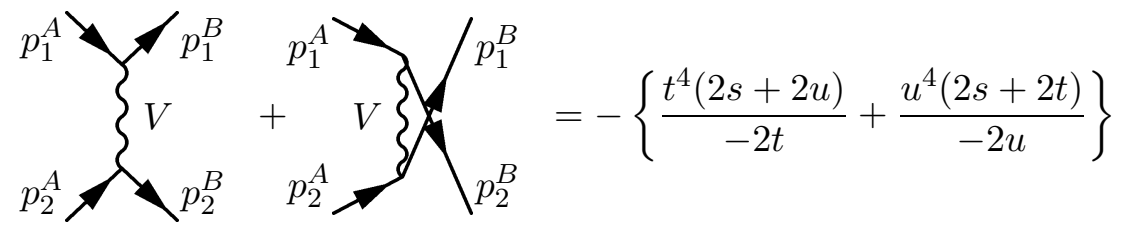

If we apply the relation $s-t-u=0$ the quartic and exchange contributions exactly cancel.

Note that the $C$ and $D$ fields do not contribute at either $\Lambda_{5}$ (scalar scattering through scalar exchange) or at $\Lambda_{4}$ (scalar scattering through vector exchange). So even if we truncated the theory at the level at the first massive mode, the strong coupling scale would already be $\left(m_{1}^{2} M_{\mathrm{Pl}}\right)^{1 / 3}$. However, to see the cancellation of the remaining diagrams, we need to include the effects of the heavier fields.

It is straightforward to work through remaining scattering processes. The computations

are more involved, but at tree-level all the amplitudes involving the Lagrangian (B.17) are exactly zero.

\section{References}

[1] N. Arkani-Hamed, H. Georgi and M. D. Schwartz, arXiv:hep-th/0210184.

[2] N. Arkani-Hamed and M. D. Schwartz, arXiv:hep-th/0302110.

[3] M. Fierz and W. Pauli, Proc. Roy. Soc. Lond. A 173, 211 (1939).

[4] A. Karch and L. Randall, JHEP 0105, 008 (2001) [arXiv:hep-th/0011156].

[5] M. D. Schwartz, Phys. Lett. B 502, 223 (2001) [arXiv:hep-th/0011177].

[6] N. Arkani-Hamed, A. G. Cohen and H. Georgi, "(De)constructing dimensions," Phys. Rev. Lett. 86, 4757 (2001) [arXiv:hep-th/0104005].

[7] C. T. Hill, S. Pokorski and J. Wang, Phys. Rev. D 64, 105005 (2001) [arXiv:hep-th/0104035].

[8] N. Arkani-Hamed, A. G. Cohen and H. Georgi, Phys. Lett. B 513, 232 (2001) [arXiv:hep-ph/0105239].

[9] R. S. Chivukula and H. J. He, Phys. Lett. B 532, 121 (2002) [arXiv:hep-ph/0201164].

[10] R. Sekhar Chivukula, D. A. Dicus and H. J. He, Phys. Lett. B 525, 175 (2002) [arXiv:hep-ph/0111016]. 